\title{
Cartan approach to Teleparallel Equivalent to General Relativity: a review
}

\author{
E. Huguet ${ }^{1}$, M. Le Delliou ${ }^{2,3}$, and M. Fontanini ${ }^{1}$ \\ 1 - Université de Paris, APC-Astroparticule et Cosmologie (UMR-CNRS 7164), F-75006 Paris, France.* \\ 2 - Institute of Theoretical Physics, School of Physical Science and Technology, \\ Lanzhou University, No.222, South Tianshui Road, Lanzhou, Gansu 730000, P $R$ China and \\ 3 - Instituto de Astrofísica e Ciências do Espaço, Universidade de Lisboa, \\ Faculdade de Ciências, Ed. C8, Campo Grande, 1769-016 Lisboa, Portugal ${ }^{\dagger}$
}

(Dated: January 19, 2021)

\begin{abstract}
In previous works, questioning the mathematical nature of the connection in the translations gauge theory formulation of Teleparallel Equivalent to General Relativity (TEGR) Theory led us to propose a new formulation using a Cartan connection. In this review, we summarize the presentation of that proposal and discuss it from a gauge theoretic perspective.
\end{abstract}

PACS numbers: 04.50.-h, 11.15.-q, 02.40.-k

\section{CONTENTS}

\section{Introduction}

II. Why a Cartan connection to describe TEGR?
A. Constitutive elements for gauge-TEGR
B. Specific features of gauge TEGR connection

III. Cartan TEGR
A. The Cartan connection
1. Klein geometry
2. Cartan geometry
3. The TEGR connection
B. Matter coupling with Cartan connection
1. From Cartan connection to Levi-Civita coupling

2. A remark about the gravitational field in Cartan TEGR

C. Is Cartan TEGR a gauge theory? Frames as gauge fields

IV. A note on Cartan GR

V. Conclusion

Acknowledgements

A. Right (left) G-space, homogeneous space and coset space $\mathrm{G} / \mathrm{H}$

B. A note on gravity as a gauge theory of translations: a not so obvious relation

References

\section{INTRODUCTION}

1 As a description of gravity, the Teleparallel Equivalent to General Relativity (TEGR) offers a symmetric and

2 classically equivalent way of presenting the physics described by General Relativity (GR): the main difference in perspective being that GR uses curvature of spacetime to describe the effects of gravity requiring zero torsion, while TEGR requires zero spacetime curvature and fully encodes gravity in the torsional part. This mirror approach to the description of gravity is not proprietary to TEGR, Symmetric Teleparallel Gravity (STGR), introduced in Ref. [1], is another example of a possible equivalent description where curvature and torsion are symmetrically taken to be null and non-metricity contains all the relevant physics. In the many years TEGR has been around, its formal presentation has taken various forms, from the translation-gauge approach of Ref. [2], the "pure tetrads formalism" point of view in Ref. [3], to the tensorial formalism of Ref. [4]. Its alternative view of gravity also created fertile ground for the proposal of naturally ghost free modified gravity developments such as $f(T)[5-7], f(T, B)[8], f(R, T)[9]$, or other generalisations such as Lovelock Teleparallel Equivalent Gravity [10], or Conformal TEGR [3, 11, 12].

One of the advantages presented by TEGR over GR is the claim that it can be formulated as a gauge theory for the translation group [see 2, 13, and references herein]; in such formulation the principal bundle framework of gauge theories is paralleled to extract the TEGR torsion from the curvature of a connection defined on the principal bundle of translations. In this approach, the socalled canonical one-form, required to define the torsion, is indeed implicitly identified with the connection oneform. Since the definition of the canonical one form, as a structure element of the frame bundle, does not fit with that of a connection, this identification becomes problematic [14-16]. The Cartan connection has therefore been proposed as a solution to this difficulty, it has in fact the right geometrical properties while being able to offer a bundle description and thus possibly support a gauge theory interpretation. This paper reviews such proposal,

\footnotetext{
*michele.fontanini@gmail.comhuguet@apc.univ-paris7.fr

† (delliou@lzu.edu.cn,)morgan.ledelliou.ift@gmail.com
} 
its recent developments, as well as additional details on related topics.

Differential geometry definitions and concepts can be referred to in [17-20]. In Sec. II, we will overview the requirements emerging from TEGR that would be necessary to express it in gauge form. A review of the coherent framework using a Cartan connection for TEGR, with its possible opening to the gauge point of view, is spelled out in Sec. III, followed, in Sec. IV, by a small note on such framework's application to GR, before concluding in Sec. V.

\section{WHY A CARTAN CONNECTION TO DESCRIBE TEGR?}

\section{A. Constitutive elements for gauge-TEGR}

In the context of TEGR and gauge theory, let us examine how the constitutive elements of TEGR fit within the framework of a typical gauge theory, as found in particle physics.

On physical side, the main constitutive elements of such theories are gauge fields and their associated field strengths. They are dynamical fields whose free field equation (uncoupled to matter) exhibits gauge invariance. They mediate an interaction between matter fields and ensure that the matter field equations are locally invariant under some symmetry. On the mathematical side, the gauge fields are sections of Ehresmann connections defined on a principal $G$-bundle, $G$ being a the global symmetry group of the free matter-field equations. The field strengths are (sections of) the curvature of these connection one-forms.

In TEGR, spacetime is a metric manifold $(M, g)$, gravity is carried by torsion, curvature is null, as well as nonmetricity. Taking the Cartan view, the metric is induced by orthonormal (co-)frames (tetrads) through $\eta(e, e)=g$, $\eta$ being the metric of Minkowski tangent space. Here we recall some central structures related to these notions:

1. the orthonormal frame bundle $O M$, a principal $\mathrm{SO}(1,3)$-bundle, sub-bundle of the frame bundle $F M$ : its base manifold is the spacetime and its fibers contains all the orthonormal frames $e$ at a base manifold point, each frame being in one-toone correspondence with an element of $\mathrm{SO}(1,3)$ a (local) Lorentz transformation,

2. the canonical one-form $\theta$, an $\mathbb{R}^{4}$-valued one-form, defined through:

$$
(\theta(e), V)=\left(e^{-1}, \pi_{*} V\right),
$$

or in coordinates,

$$
\theta^{a}(e)[V]=e^{a}\left[\pi_{*} V\right]=V^{a}
$$

where $e$ is a frame at a point $x$ of the base manifold $M, e^{-1}$ its co-frame, $V$ a vector field of the tangent bundle $T O M$, and $\pi$ the projection on the base. The canonical one-form and the co-frames are related through the important equality

$$
\sigma^{*} \theta=e,
$$

where $\sigma$ is some section of the frame bundle ${ }^{1}$.

3. an Ehresmann connection $\omega$ (a Lorentz or spin connection in this context) defined on $O M$ allowing us to define the torsion through the usual expression on the frame bundle:

$$
\Theta(\omega)=d \theta+\omega \wedge \theta
$$

The two objects $O M$ and $\theta$ are defined as soon as a spacetime is present. If in addition an Ehresmann connection is defined on the frame bundle, these structures allow us to define the torsion through Eq. (3). Along some section $\sigma$, this torsion leads to the torsion on the spacetime (base manifold): $T=\sigma^{*} \Theta=d e+\widetilde{\omega} \wedge e$, where $\widetilde{\omega}=\sigma^{*} \omega$.

A first observation is that the canonical one-form, the frame bundle (on which it is defined) and a (Lorentz) connection are needed to obtain the torsion. By contrast, the curvature, which is defined through the expression: $\Omega(\omega)=d \omega+\omega \wedge \omega$, can be defined as soon as an Ehresmann connection $\omega$ is defined on a principal $G$-bundle, as is the case for particle physics gauge theories. A second observation is that in the TEGR framework the curvature of the connection is by definition zero, which singles out the Weitzenböck connection: $\omega_{W}$.

From these considerations, we notice that the identification of the basic objects of TEGR with those of a gauge theory are far from obvious: although a principal bundle (the frame bundle) is present, the connection naturally associated with TEGR has a null curvature, and the canonical one-form (entering in the definition of torsion) possesses no equivalent in the usual gauge theoretic framework.

A way out this issue is to consider on physical grounds that, since in TEGR gravity manifests itself through torsion, this torsion should be considered as the field strength of a yet undetermined gauge theory. A first step in finding a gauge formulation of TEGR can then be made by setting a connection, defined on the orthonormal frame bundle (or a principal bundle containing it), whose torsion is the curvature.

\section{B. Specific features of gauge TEGR connection}

If torsion, as defined in Eq. (3), needs to be the curvature of some connection $\omega_{C}$, the sought for connection re-

\footnotetext{
1 Note that, since $\theta$ is an element of structure this relation essentially states an equivalence between local sections and local frames. A change of gauge, which in the bundle formalism of gauge theories is a change of section, here precisely corresponds to a change of frame.
} 
quires to be built out of both the Ehresmann connection $\omega$ appearing in the torsion and the canonical one-form $\theta$. These two objects $\omega$ and $\theta$ are different in nature. Let us briefly recall some of their properties, to finally motivate the appearance of a Cartan connection.

The one-form $\omega$ defines an Ehresmann connection in the principal $\mathrm{SO}(1,3)$-bundle $O M$. In a general sense, an Ehresmann connection is a mean to split in a unique way each tangent spaces of a fiber bundle into vertical and horizontal subspaces. As vertical subspaces are always defined as tangent spaces to the fibers, ${ }^{2}$ the Ehresmann connection uniquely specifies horizontal subspaces and, in this sense, defines uniquely the notion of horizontality in the fiber bundle. To be meaningful in a principal $G$-bundle, the splitting between vertical and horizontal subspaces must be invariant under the action of the symmetry group $G$. This invariant splitting is realized in practice through a connection one-form $\omega_{E}$ such that:

1. it takes its values in the Lie algebra $\mathfrak{g}$ of the Lie group $G$,

2. it satisfies: $R_{g}^{*} \omega_{E}=A d_{g^{-1}} \omega_{E}, R_{g}$ being the right action of $G$ on the bundle,

3. it reduces to the Maurer-Cartan form $\omega_{G}$ of the group $G$ along the fibers: $\omega_{E}(V)=\omega_{G}(V)$, for any vertical vector $V$.

Following these properties, at each point of the $G$-bundle, vertical vectors are mapped to the Lie algebra $\mathfrak{g}$ and horizontal vectors belong to the kernel of $\omega_{E}$. In addition, an Ehresmann connection allows one to construct parallel transport and its related covariant derivative. ${ }^{3}$

The one-form $\theta$ Eq. (1), contrary to a connection oneform, is canonically and only defined in the frame bundle (hence on its restriction $O M$ ). The content of Eq. (1) can be described as follows: for each frame bundle point, mapped to coordinates $(e, x)$, the action of $\theta$, through each of its components $\theta^{a}$, consists in mapping a bundle's tangent space vector $V$ to its corresponding component $V^{a}$ in the frame $e$ at the base manifold tangent space point $x$. In comparison with the Ehresmann connection, $\theta$ :

1. takes its values in $\mathbb{R}^{4}$,

2. satisfies: $R_{g}^{*} \theta=g^{-1} \theta, R_{g}$ being the right action of the matrix group $\mathrm{GL}(4, \mathbb{R})$ on $F M$ (or $\mathrm{SO}(1,3)$ on $O M)$,

\footnotetext{
2 Note that since the fiber is isomorphic to the group in a Principal G-bundle, the tangent spaces to fibers are all isomorphic to the Lie algebra $\mathfrak{g}$.

3 The relation between these notions appears more evidently in the frame bundle, where a parallel-transported frame (so-called autoparallel frame field) along a base manifold curve $\tilde{\gamma}$ induces a horizontal curve $\gamma$, i.e. whose tangent vector is always horizontal, in the full bundle [see for instance 17, p. 544 19.5.1]
}

3. is horizontal ${ }^{4}$ in the sense that: $\theta(V)=0$, for any vertical vector $V$.

As is physically motivated, a connection promotes a global symmetry to a local symmetry. At the mathematical level, this local symmetry is encoded in the connection's values taken in Lie algebra of the symmetry group. Thus, the orthonormal frame bundle OM's Ehresmann connection $\omega$ takes values in the Lie algebra of the Lorentz group. Similarly, the TEGR connection $\omega_{C}$ we are seeking, with torsion as field strength, should implement local symmetry as well as include the additional $\theta$ one-form. Since $\theta$ takes its values in $\mathbb{R}^{4}$, which can be considered as the algebra for the translation group, this suggests to build an $\omega_{C}$ with values in the algebra of the Poincaré group $\mathrm{SO}(1,3) \rtimes \mathbb{R}^{4}$.

The considerations above would appear to point towards a gauge theory with an affine connection defined on the principal Poincaré bundle, an affine bundle denoted hereafter $P M$. However, starting from the orthonormal frame bundle, it suffices to enlarge its Lorentz connection's algebra, so we will consider a more minimalistic generalization: a Cartan connection, valued in the Poincaré algebra while being defined on the original orthonormal frame bundle $O M$.

\section{CARTAN TEGR}

\section{A. The Cartan connection}

A Cartan connection enters in the definition of a Cartan geometry which can either be considered to generalise Riemann geometry or Klein geometry, each themselves offering a generalization of Euclid geometry. ${ }^{5}$ An introduction to Cartan geometries in the context of gravity may be found in [21, 22], while a comprehensive mathematical account is given in [23]. Since Riemann geometry is well known in GR, let us briefly discuss the notion of Klein geometry, which is as important as Riemann's, for the Cartan generalization.

\section{Klein geometry}

Instead of introducing space curvature to get away from Euclid geometry, as in Riemann geometry, Klein geometry generalizes Euclid spaces to homogeneous spaces (also termed maximally symmetric, see App. A for a reminder). The latter are always isomorphic (and consequently identified) to some coset space $G / H$ (also termed

\footnotetext{
${ }^{4}$ We emphasize that $\theta$ is defined independently from the presence of a connection, which is not responsible here for the horizontality.

5 These generalizations applies, when a metric is present, to positive definite metric (e.g. Riemannian geometry) or to nonpositive definite metric (pseudo-Riemannian geometry).
} 
quotient space), where $G$ is a Lie group and $H$ one of its (closed) subgroup. This basically defines a Klein geometry $(G, H)$, which is the homogeneous space $G / H$. When $G / H$ is connected, a connected Klein geometry is obtained - disconnected homogeneous spaces are irrelevant for Cartan geometry, so we'll focus on connected ones. In this context the familiar three dimensional Euclidean space appears as a particular case of Klein geometry where $G=\mathrm{SO}(3) \rtimes \mathbb{R}^{3}$ and $H=\mathrm{SO}(3)$ the rotation group.

As they derive from the action of Lie groups on (differentiable) manifolds (see App. A), homogeneous spaces are (differentiable) manifolds ${ }^{6}$. In four dimensions, Lorentzian homogeneous spaces are the familiar spaces of:

$$
\begin{aligned}
& \text { Minkowski: } G=\mathrm{SO}(1,3) \rtimes \mathbb{R}^{4}, H=\mathrm{SO}(1,3) . \\
& \text { de Sitter: } G=\mathrm{SO}(1,4) H=\mathrm{SO}(1,3) . \\
& \text { Anti-de Sitter: } G=\mathrm{SO}(2,3), H=\mathrm{SO}(1,3) .
\end{aligned}
$$

Many properties of homogeneous spaces are inherited from the Lie groups underlying their structures. An important case is the relation between their tangent spaces and the Lie algebras of the group $\mathrm{G}$ and of its subgoup $\mathrm{H}$ : the Lie group $G$ can itself be seen as principal fiber bundle of fiber $H$, with the homogeneous space $G / H$ as base manifold, i.e. $\mathrm{G}(\mathrm{G} / \mathrm{H}, \mathrm{H})$ [see for instance 20, p. 55]. The adjoint representation of $H$ (the action of $H$ on its Lie algebra) allows one to associate a vector bundle, with fiber $\mathfrak{g} / \mathfrak{h}$, to $\mathrm{G}(\mathrm{G} / \mathrm{H}, \mathrm{H})$. This associated bundle can be shown to be isomorphic to the tangent bundle of the homogeneous space $G / H, T(G / H)$ [see 23, prop. 5.1 p. 163 , for details]. This important property means, in particular, that each tangent space to a homogeneous space is isomorphic to the quotient $\mathfrak{g} / \mathfrak{h}$, namely that at each point $x$ of $G / H$,

$$
T_{x}(G / H) \simeq \mathfrak{g} / \mathfrak{h}
$$

In addition to the above relation, the tangent spaces inherit, from the aforementioned bundle isomorphism, all the structures defined on $\mathfrak{g} / \mathfrak{h}$. In particular a nondegenerate $H$-invariant metric defined on $\mathfrak{g} / \mathfrak{h}$ pulls-back to an $H$-invariant metric on the corresponding homogeneous space. A paradigmatic example in GR is given by the metric of the four dimensional homogeneous (maximally symmetric) spacetimes of Minkowski, de Sitter and Anti-de Sitter [21], inherited from the symmetries of the corresponding coset spaces.

\footnotetext{
${ }^{6}$ Here, the hypothesis that $H$ is a (topologically) closed subgroup of the Lie group $G$ is central for the space $G / H$ to be a manifold [see for instance 23, p. 146].
}

\section{Cartan geometry}

We introduced Sec. III A recalling that Cartan geometry represents at the same time a generalization of Klein and Riemann geometries. Here we will give more details on how it actually does merge both. In Sec. III A 1, we discussed how Klein geometry generalizes Euclid by considering the Euclidean space as a particular case of homogeneous spaces, and how these spaces are defined by the quotient of a Lie group $G$ by one of its (closed) subgroups $H$, sharing the symmetries of the group $G$. Riemann geometry is historically an $N$-dimensional generalisation of the two-dimensional Gaussian differential geometry of surfaces departing from the Euclid (plane) geometry by introducing curvature. In contemporary terminology Riemann geometries are metric orientable differentiable manifolds, equipped with the Levi-Civita connection, their curvature measures how they locally depart from the flatness of their Euclidean tangent spaces, while the connection relates tangent spaces at different points.

In Cartan geometry the Euclidean tangent space is generalized to a tangent homogeneous space. To simplify visualisation of such tangent spaces, the two-dimensional case can be examined: the Riemann view considers a tangent plane at each point of the surface, its contact point with the surface constituting its origin, when identified with the (invariant) vector space $\mathbb{R}^{2}$ (under the rotation group $\mathrm{SO}(2)$ ). By contrast, the Cartan view rolls, without slipping, a unique space on the surface, a space which can either be a plane, sphere or hyperboloid, one of the three homogeneous spaces in two dimensions. The contact point thus moves in the homogeneous space, putting in evidence its affine nature. The case of a plane therefore identifies with the affine plane $\mathbb{R}^{2}$, invariant under the affine group $\mathrm{SO}(2) \rtimes \mathbb{R}^{2}$.

These two different descriptions of tangent spaces are reflected respectively in the Cartan and Levi-Civita (generalised to affine Ehresmann) connections. They both provide methods of transfering objects between points joined by some (continuous) path $\gamma$. The Cartan connection effects the transfer of contact point, rolling without slipping, in the homogeneous space, while the Levi-Civita connection provides a rule to transfer objects along the path $\gamma$ from one plane to another.

The defining properties of a Cartan geometry are gathered in the following definitions:

A Cartan geometry, modeled over a Klein geometry $(\mathrm{G}, \mathrm{H})$, is a principal $H$-bundle $P(M, H)$ equipped with a Cartan connection.

A Cartan connection is defined through a one-form $\omega_{C}$ such that:

1. it takes values in the algebra $\mathfrak{g} \supset \mathfrak{h}$ of $G \supset H$.

2. it is, at each point $p$ of the $H$-bundle, a linear isomorphism between the tangent space $T_{p} P$ at $p$ and the Lie algebra $\mathfrak{g}$. This property re- 
quires that $G$ and the tangent space $T_{p} P$ share the same dimension.

3. it satisfies: $R_{h}^{*} \omega_{C}=A d_{h^{-1}} \omega_{C}, R_{h}$ being the right action of $H$ on the bundle,

4. it reduces to the Maurer-Cartan form $\omega_{H}$ of the group $H$ along the fibers.

The Cartan geometry definition sets its own mathematical framework. In the TEGR situation we are interested in, the $H$-bundle will be the orthonormal frame bundle $O M$ with $H=\mathrm{SO}(1,3)$ and $M$, the spacetime. In accordance with the conclusion of Sec. II B, we will take, for the group $G$, the Poincaré group and, consequently, for the homogeneous space (the Klein geometry), we will use the Minkowski space.

The case we are interested in then presents an additional property: its Cartan geometry is reductive. Formally [see 23, p. 197, for details], this means that the Lie algebra decomposition as vector space $\mathfrak{g}=\mathfrak{h} \oplus \mathfrak{g} / \mathfrak{h}$ is $\operatorname{Ad}(H)$-invariant. In practice, among other results, it implies that any $\mathfrak{g}$-valued form defined on $P$ splits along this decomposition and, in particular, the connection oneform $\omega_{C}$ splits into $\mathfrak{h}$ and $\mathfrak{g} / \mathfrak{h}$ parts. For the Poincaré algebra, these correspond respectively to the Lorentz and translation parts, $\mathfrak{h}=\mathfrak{s o}(1,3)$ and $\mathfrak{g} / \mathfrak{h}=\mathbb{R}^{4}$. Consequently, the reductive Cartan connection one-form splits into

$$
\omega_{C}=\omega+\theta
$$

where $\omega$ is an $\mathfrak{h}$-valued Ehresmann type connection oneform, and $\theta$ a $\mathfrak{g} / \mathfrak{h}$-valued one-form, both defined on the principal $H$-bundle. This decomposition also follows for the curvature of $\omega_{C}$,

$$
\Omega\left(\omega_{C}\right)=\Omega(\omega)+\Theta(\omega)
$$

where the curvature of the $\omega$ part reads $\Omega(\omega)=d \omega+\omega \wedge \omega$ and both parts combine in the torsion $\Theta(\omega)=d \theta+\omega \wedge \theta$.

The definition of the Cartan connection, through $\omega_{C}$, differs from that of the Ehresmann connection, in Sec. II B, in the first two properties which we will now discuss.

From property 1, instead of being valued in the Lie algebra of the fiber $\mathfrak{h}, \omega_{C}$ is valued in the whole $\mathfrak{g}$ algebra. This agrees with the "minimal extension" mentioned at the end of Sec. II B since, of the full algebra $\mathfrak{g}$, only appears the translations part $\mathfrak{g} / \mathfrak{h}$.

Property 2 relies both on the Klein geometry and the soldering property. It imposes the tangent space, $T_{p} P$ of $P(M, H, \pi)$ at $p$, and $\mathfrak{g}$ to be isomorphic. In addition, its vertical part is always defined as the tangent space to the fiber and is also isomorphic to $\mathfrak{h}$; Eq. (4) implies that the orthogonal complement $\mathfrak{g} / \mathfrak{h}$ of $\mathfrak{h}$ in the decomposition $\mathfrak{g}=\mathfrak{h} \oplus \mathfrak{g} / \mathfrak{h}$ identifies with the tangent space $T_{\pi(p)}(G / H)$ to the homogeneous space at $\pi(p)$; therefore, it can be identified with the tangent space $T_{\pi(p)}(M)$ to the base manifold at $\pi(p)$. In the same way as $T_{\pi(p)}(M)$ is a fiber of the tangent bundle $T M, T_{\pi(p)}(G / H)$ is the fiber of a "tangent homogeneous-space" bundle of $M$ associated to $P: P \times_{H} G / H$. Since the connection induces $\mathfrak{g} / \mathfrak{h} \simeq$ $T_{\pi(p)}(G / H) \simeq T_{\pi(p)}(M)$, that identifies a tangent space of $M$ to a tangent space of a fiber of $P \times_{H} G / H$, it thus effects a soldering [see for instance 14, App. 3] of the $H$ principal bundle to its base $M$, illustrating this "built-in property" of the Cartan connection.

Finally, properties 3 and 4 correspond identically to properties 2 and 3 for Ehresmann connection one-forms.

Returning to the frame bundle we consider for TEGR (with its Cartan connection), and as anticipated in Eq. (5) notation, its canonical one-form $\theta$ does indeed correspond to the $\mathfrak{g} / \mathfrak{h}$ term in the Cartan connection. The interpretation of the Cartan geometry as a movement of the tangent space rolling without slipping on the base, added with Eq. (2), allows us to interpret that term of the Cartan connection in link with the so-called moving (co)frame.

\section{The TEGR connection}

From the discussion above and the requirement that for TEGR the curvature of the connection yields the torsion, Eq. (5) specializes to

$$
\omega_{C}=\omega_{W}+\theta
$$

where the Ehressman term, $\omega_{W}$, stands for the curvatureless Weitzenböck connection and where $\theta$ coincides with the canonical one-form on $O M$. Then, through Eq. (6) or by direct calculation [see 16], the required relation $\Omega\left(\omega_{C}\right)=\Theta\left(\omega_{W}\right)$ is obtained.

As we previously pointed out while analyzing their differences, the Cartan connection, that we suggest describes TEGR, contrasts with the Ehresmann connection, that represents particle physics gauge theories. At this stage it is premature to claim whether this framework allows TEGR to be seen as a gauge theory, however, in the Cartan geometry, $\omega_{C}$ does give the expected field strength, in addition to relating gravitation to the translation symmetry, in accordance with Noether's theorem: while each term of $\omega_{C}$ in Eq. (7) corresponds to Lorentz and translation symmetries respectively - through values in the Poincaré Lie algebra - and as the Weitzenböck curvature vanishes, the only contributing curvature (field strength) term comes from the translation $\left(\mathfrak{g} / \mathfrak{h}=\mathbb{R}^{4}\right)$ valued $\theta$ term. In contrast with the difficulties to extract a translation term from the usual formulation of GR (see App. B), the natural appearance of translation with $\theta$ in Cartan TEGR counts as its achievement. Furthermore, to preserve the physical relation between field strength and gauge field in the Cartan TEGR framework, Eq. (2) points toward identifying the co-frame as the gauge field of the theory. To settle the identification of a gauge field in the theory requires to examine the coupling to matter, since particle physics coupling is mediated by the gauge field. 


\section{B. Matter coupling with Cartan connection}

In particle physics gauge theories, charged matter fields interact through the exchange of gauge bosons, mediating the interaction. At the classical level, this interaction is described thanks to a covariant derivative ensuring the minimal coupling. That covariant derivative is directly related to an Ehresmann connection whose pullback on the base manifold (the Minkowski space) is the gauge field. In fact, covariant derivative and parallel transport are directly built from an Ehresmann connection in a principal bundle. In classical GR the coupling to classical matter field is also realized through a covariant derivative: in Cartan (tetrads) formalism, the covariant derivative is implemented from the Levi-Civita connection one-form $\omega_{L C}$ in the frame bundle. So far, GR - and thus the Levi-Civita connection - reproduces dramatically well observational data ${ }^{7}$ and hence compels any formulation of gravity to reproduce the Levi-Civita coupling.

\section{From Cartan connection to Levi-Civita coupling}

In this section we will recall from [24] how to obtain the Levi-Civita part of coupling to matter from the Cartan connection one-form $\omega_{C}$, proposed to describe TEGR.

Starting from the Cartan geometry, the Levi-Civita coupling is obtained in three main steps, commented hereafter

1. The Cartan connection one-form $\omega_{C}$ on $O M$ is mapped to an Ehresmann connection one-form $\omega_{E}$ thanks to the Sharpe theorem [Prop. 3.1 p. 365 of 23] (also reproduced in [24]).

2. The Weitznböck term, present in $\omega_{E}$ : the first term of the r.h.s. of Eq. (7), is mapped to the LeviCivita one-form thanks to the contorsion one-form defined on $O M$ [25, theorem 6.2 .5 p. 79].

3. The resulting one-form, an affine connection on the Poincaré principal bundle $P M$, is then pulled back on $O M$ with a map [20, proposition 3.1 p. 127] that splits it into the canonical and the Levi-Civita oneforms, parallel transport and covariant derivative proceeding then from the Levi-Civita Ehresmann connection, now in a Cartan (tetrads) formalism setting.

More explicitly: as the Cartan connection does not define canonically parallel transport, by contrast with Ehresmann connection, the first step relates, in our peculiar case, the set of $\omega_{C}$ to the set of affine Ehresmann

\footnotetext{
7 Unfortunately, the interpretation of the Levi-Civita connection as a gauge field mediating gravitation is not satisfying (see App. B).
}

connections on the affine (Poincaré) bundle, as obtained in [24] with the help of Sharpe theorem ${ }^{8}$. As the theorem, in our case, results in the identity of the connection oneform on the base manifold (the spacetime), the pullback of the Weitzenböck term $\omega_{W}$ in Eq. (7) is still present. To obtain the correct matter coupling requires to map this term to the Levi-Civita one-form $\omega_{L C}$.

This is done, in the second step, applying the theorem ${ }^{9}$ that relates any Ehresmann one-form from the orthonormal frame bundle $O M$ to the Levi-Civita one-form via its contorsion one-form.

Two remarks are in order here. First, although any two Ehresmann connections are always related by some specific one-form, the existence of the contorsion, and hence getting the Levi-Civita one-form, relies on the existence of a metric. That metric is present in $O M$, where the possibility to map Weitzenböck to Levi-Civita one-forms can be traced back to the choice of the Minkowski metric for tangent spaces to the base (the spacetime), and in this sense related to the Equivalence Principle. The second remark concerns the building of the Levi-Civita one-form in this context: as contorsion is composed from torsion, itself built out of the Weitzenböck connection, and of the canonical one-form, the resulting Levi-Civita one-form is a function of the Weitzenböck one-form, the canonical one-form, and its derivatives.

This Weitzenböck-to-Levi-Civita mapping allows us to redefine the Ehresmann one-form $\omega_{E}$, into $\widetilde{\omega}_{E}$, now the sum of a linear, Levi-Civita one-form, $\omega_{L C}$, and a translation term, the canonical one form $\theta$, also defined on the affine bundle $P M$. The third step maps back $\widetilde{\omega}_{E}$ into $O M$ [20, proposition 3.1 p. 127], where it splits into $\omega_{L C}$, and $\theta$.

Compositing these steps, the final result yields a map on $O M$ from Cartan to Levi-Civita connection:

$$
\omega_{C} \longmapsto \omega_{E}=\omega_{W}+\theta \longmapsto \widetilde{\omega}_{E}=\omega_{L C}+\theta \longmapsto\left(\omega_{L C}, \theta\right) .
$$

This map provides matter coupling to TEGR-gravity in agreement with the familiar coupling of GR, as well as the Fock-Ivanenko derivative in Cartan (tetrads) formalism.

\section{A remark about the gravitational field in Cartan TEGR}

In classical GR, the gravitational interaction is carried by the metric $g$ or, in Cartan (tetrads) formalism, by the frame field $e$. The metric, or the frame field, also entirely determines the Levi-Civita connection. Although the Cartan TEGR framework reaches these results from an entirely different perspective, $e$ remains the dynamical

\footnotetext{
${ }^{8}$ A technical condition restricts the set of possible Ehresmann connections. However, since it bears no influence on our results, we just point this out to the interested reader.

9 The proof in the bundle formalism is given in [25, theorem 6.2.5 p. 79].
} 
field and coupling remains mediated through the LeviCivita connection. The gravitational field thus remains carried by the frame field in Cartan TEGR.

\section{Is Cartan TEGR a gauge theory?}

As discussed Sec. II A, TEGR requires some crucial structural elements that are absent from particle physics gauge theories. Therefore, adopting the usual particle physics structure as gauge theory definition would exclude TEGR. In the wider context of gauge-gravity theories [see 26 , for a comprehensive, historical account] some rather deep change of paradigm were considered [27]. Here, following [24] on physical grounds, we only minimally propose to extend the usual gauge theory framework to include the required extra structure for TEGR. Although this extension hints at a possible interpretation of Cartan TEGR as a gauge theory, which we discuss below, we do not claim adhesion to it, leaving interpretation open.

Frames as gauge fields

The Cartan connection $\omega_{C}$, as decomposed in Eq. (7), yields a curvature equal to the torsion. Moreover, since the curvature of the Weitzenböck connection vanishes, the only contributing dynamical field to the field strength remains the field of frames. This, as pointed out in Sec. III A 3, hints at the field of frames behaving as a gauge field. We also pointed out Sec. III B 2 that the frame can be considered as mediating the gravitational field. This is already the case in GR since the Levi-Civita connection, determined from the metric, thus the frames, in a Cartan (tetrads) formulation, mediates coupling to tensorial and spinorial fields. Additionally, since classical scalar fields also couple to gravity, while their covariant derivative reduces to a partial derivative, coupling should be present in the partial derivative. As the tangent vector $\partial_{\mu}$ decomposes in the frame basis $e_{a}$ following $\partial_{\mu}=\left(\partial_{\mu}\right)^{a} e_{a}$, such coupling reinforces the interpretation of the frame field as mediating gravitational interaction.

All the above arguments advocate for the interpretation of $e$ as the gauge field on the basis that it mediates interaction. At the same time, interpreting $e$ as a gauge field departs from the paradigmatic framework of gauge theories, that would relate $e$ to an Ehresmann connection by a simple pullback on the base manifold: it relates instead to the Cartan connection $\omega_{C}$ through the composite map combining Eqs. (8) and (2). To interpret TEGR as a gauge theory thus requires to abandon the strict correspondence between gauge field and Ehresmann connection. Note that the usual particle physics gauge theories remain unaffected by this change, since it only involves external ingredients.

In addition to mediating interaction, particle physics gauge fields also implement some local (i.e. spacetime point dependent) symmetry invariance in matter field equations. As a frame $e$, from Eq. (2), is clearly related to the canonical one-form $\theta$, valued in the translation part of the Poincaré algebra, it thus relates to local and infinitesimal translations. The discussion above reveals that matter coupling involves two occurrences of the frame $e$ : in the expansion of the partial derivative and in the Levi-Civita connection, seen as the difference between the non-dynamical Weitzenböck connection and the frame "induced" contorsion. However, the field $e$ does not emerge from localisation of a global translation symmetry. Instead, the symmetry is already local and infinitesimal since $e$ only relates to the translation algebra. The usual process involved in "gauging a theory" by which a globally invariant matter field Lagrangian becomes locally invariant with the introduction of the gauge field in the derivatives is not present in this context. Moreover, the basic symmetry of GR, i.e. diffeomorphism invariance (the invariance under spacetime coordinate changes), does not proceed from translation symmetry but is already a built-in property of differential geometry.

From the above discussion, the frame $e$, on the basis that it mediates the interaction with matter fields, can be perceived to share physical properties of a gauge field. Nevertheless, such an interpretation requires to enlarge the notion of gauge field compared with its meaning in usual particle field theories. In particular, the frame field $e$ is the pullback of the canonical one-form, rather than that of an Ehresmann connection, and is the translation part of a Cartan connection whose curvature, still considered as the field strength, is the torsion.

\section{A NOTE ON CARTAN GR}

As TEGR is equivalent to GR, the Cartan TEGR structure to represent TEGR can be transposed to use a Cartan connection to describe GR. This transposition can be obtained by

1. setting the Ehresmann term in the Cartan connection one-form (5) to the Levi-Civita connection: $\omega_{C}=\omega_{L C}+\theta$,

2. recognising the mapping using contortion reduces to identity, as torsion - and thus contorsion - vanishes for the Levi-Civita connection, such that the map (8) becomes

$$
\omega_{C} \longmapsto \omega_{E}=\omega_{L C}+\theta \longmapsto\left(\omega_{L C}, \theta\right) .
$$

The Cartan curvature of the corresponding $\omega_{C}$ then reduces to the Riemann curvature term of the Levi-Civita connection, as expected in GR. Note that the parallel transport for such reductive Cartan connection could be directly seen as induced, in the initial bundle, by the Ehresmann part of its one-form $\omega_{C}$. The only difference 
of this framework with the usual Cartan (tetrad) formalism of GR lies in the Cartan connection and the possibility it awards GR, in a similar way as for TEGR above, to be conceivably interpreted as a gauge theory. Similar modifications to particle physics type gauge theory are required, in the lines described above. The possible interpretation of the frame field as a gauge field for translations could follow similar arguments to the ones presented above, leading to a recognition of the resulting Cartan GR as a gauge theory to be similarly left open.

We note that the use of Cartan connection to describe GR has already been studied, along other lines, in [22, 28].

\section{CONCLUSION}

In this paper we have reviewed our proposal formulating TEGR from a Cartan type connection. The theory we obtain, and refer to as Cartan TEGR, produces identical predictions to TEGR and thus to GR. It provides a consistent mathematical framework in which the torsion is obtained as the curvature of a reductive Cartan connection. This connection is the sum of the curvatureless Weitzenböck connection and the so-called canonical oneform whose pullback along some section on spacetime is a frame. The usual (GR) coupling to matter fields is obtained thanks to Sharpe's theorem relating Cartan and Ehresmann connections, and to the introduction of the contorsion one-form in relation with the equivalence principle.

Cartan TEGR is then examined from a gauge theoretic perspective. This is done without claiming it is a gauge theory - this interpretation is left open - and we discuss the mandatory extensions and the departures from the particle physics gauge theory paradigm. This perspective allows us to specify to which extent the (local) field of frame could be recognized as a translation gauge field mediating gravity, and how it could be associated to torsion, as a field strength.

\section{ACKNOWLEDGEMENTS}

The authors wish to thanks D. Bennequin and T. Lawrence for helpful discussions, respectively, on geometry and on the status of diffeomorphisms in groups and gravity. The work of M. Le D. has been supported by Lanzhou University starting fund, and the Fundamental Research Funds for the Central Universities (Grant No.lzujbky-2019-25).

\section{Appendix A: Right (left) G-space, homogeneous space and coset space $\mathrm{G} / \mathrm{H}$}

We follow Fecko [17] (Sec. 13.1, 13.2 for details). A manifold $M$ on which a right (left) action of a Lie group
$\mathrm{G}$ is available is a right (left) G-space. The orbit of some point $x$ in $M$ is the set of points which can be reached from $x$ by the group action. Note that orbits are disjoints. In the special case when $M$ only contains a single orbit, that is when the action of $\mathrm{G}$ is transitive on $M$, the $\mathrm{G}$ space is called a homogeneous space. ${ }^{10}$

The left (right) coset space $\mathrm{G} / \mathrm{H}$, where $\mathrm{H}$ is a topologically closed subgroup of $\mathrm{G}$, is the set of equivalent classes $[g]$ for the relation noted ${ }^{11} \sim$. Since the left product in $G$ $g g^{\prime}$ is transitive, the left action in $\mathrm{G} / \mathrm{H}$ defined through $L_{g}\left[g^{\prime}\right]:=\left[g g^{\prime}\right]$ is then transitive, and consequently $\mathrm{G} / \mathrm{H}$ is a homogeneous space.

In this context, the group $\mathrm{G}$ does act, through $L_{g}$, on $\mathrm{G} / \mathrm{H}$, and therefore this coset space can be considered as a left G-space. In fact, one can show that all homogeneous G-spaces are obtained in this way, which means that any homogeneous space manifold $M$ with a group action from $\mathrm{G}$ is isomorphic to a $\mathrm{G} / \mathrm{H}$ for some closed subset $\mathrm{H}$.

Moreover, one can show that $\mathrm{H}$ can be taken as the stabilizer of some point $x$ of $M .{ }^{12}$ Since $\mathrm{M}$ is homogeneous, any two points being in the same orbit share isomorphic stabilizers and the choice of the point $x$ is unimportant.

Finally, we note that $\mathrm{G}$ is a principal $\mathrm{H}$-bundle over the (left) coset space $\mathrm{G} / \mathrm{H}$ if $\mathrm{G}$ is a Lie group and $\mathrm{H}$, a (topologically) closed (not necessarily invariant) subgroup [see for instance 20, Exemple 5.1 p.66]. Here the action of $\mathrm{H}$ on $\mathrm{G}$ is just the right multiplication. The fibers are the left cosets of $\mathrm{H}$ and since the fiber containing the identity is naturally isomorphic to $\mathrm{H}$, all fibers are isomorphic to $\mathrm{H}$.

\section{Appendix B: A note on gravity as a gauge theory of translations: a not so obvious relation}

Since Noether theorem associates translations to the stress-energy tensor, it suggests that gravity, as described by the Einstein equations of General Relativity (GR), is related to translations. Whereas translation is a global symmetry of physical equations in Minkowski space, it can become local when gravity is present. This, in turn, open the possibility that gravity arises from a gauge theory of translations, where, following the usual gauge theory construction, a gauge field is minimally coupled to the (Minkowskian) matter fields in order to obtain a matter equation locally invariant under translations. ${ }^{13}$

${ }^{10} \mathrm{G}$ transitive on $M$ means there exists for all pair of point (x, y) in $M g \in \mathrm{G}$ such that $R_{g} x=y\left(L_{g} x=y\right)$.

11 The map $\sim$ is defined by: $g_{1} \sim g_{2}$ iff there exists an $h \in \mathrm{H}$ such that $g_{1}=g_{2} h\left(g_{1}=h g_{2}\right)$.

12 The stabilizer of some point $x$ of $M$ with a group action from $\mathrm{G}$ is the subset $\mathrm{G}_{x}$ of $\mathrm{G}$ (which is indeed a subgroup) whose elements leave $x$ invariant.

13 A point of terminology: by translation we mean an element of the translation group $\left(\mathbb{R}^{4},+\right)$, often abbreviated in $\mathbb{R}^{4}$. The transla- 
Unfortunately, the situation is more complicated. A first difficulty concerns the structure of a gauge theory: contrary to the action of particle physics gauge groups, involving matter and gauge fields, translations also act on space-time (often termed "external" symmetry). This relates to the soldering property [see for instance 14, App. 3]. Since gauge theories of particle physics' standard model are built on internal groups (U(1), SU(2), etc.), they are not concerned with this property. On the contrary, a translation gauge theory should take soldering into account. This suggests to enlarge the mathematical framework of gauge-theories to include this "new" ingredient. A second difficulty stems from the symme- tries under consideration: in gauge field theory the local invariance is implemented thanks to the connection (valued in the Lie algebra of the symmetry group) appearing in the covariant derivative. In general relativity, in the Cartan (tetrads) formalism, matter coupling involves the so-called spin or Lorentz connection. As it takes its value in the Lorentz algebra $\mathfrak{s o}(1,3)$, this connection takes its source in the Lorentz group $\mathrm{SO}(1,3)$ and so isn't related to the translation group.

As a result, the encoding of the natural link made by Noether theorem between gravity an translation symmetry into a gauge theory is not a straightforward issue.
[1] J. M. Nester and H.-J. Yo, Chin. J. Phys. 37, 113 (1999), arXiv:gr-qc/9809049 [gr-qc].

[2] R. Aldrovandi and J. G. Pereira, Teleparallel Gravity: An Introduction, Vol. 173 (Springer, 2013).

[3] J. W. Maluf and F. F. Faria, Phys. Rev. D85, 027502 (2012), arXiv:1110.3095 [gr-qc].

[4] J. B. Jiménez, L. Heisenberg, and T. S. Koivisto, Universe 5, 173 (2019), arXiv:1903.06830 [hep-th].

[5] R. Ferraro and F. Fiorini, Phys. Rev. D75, 084031 (2007), arXiv:gr-qc/0610067 [gr-qc].

[6] S. Capozziello, R. D'Agostino, and O. Luongo, Int. J. Mod. Phys. D28, 1930016 (2019), arXiv:1904.01427 [gr-qc].

[7] Y.-F. Cai, S. Capozziello, M. D. Laurentis, and E. N. Saridakis, Reports on Progress in Physics 79, 106901 (2016).

[8] S. Bahamonde and S. Capozziello, Eur. Phys. J. C 77, 107 (2017), arXiv:1612.01299 [gr-qc].

[9] S. Bahamonde, C. G. Böhmer, and M. Wright, Phys. Rev. D92, 104042 (2015), arXiv:1508.05120 [gr-qc].

[10] P. González, S. Reyes, and Y. Vásquez, JCAP 07, 040 (2019), arXiv:1905.07633 [gr-qc].

[11] J. Formiga, Phys. Rev. D99, 064047 (2019), arXiv:1905.04764 [gr-qc].

[12] K. Bamba, S. D. Odintsov, and D. SáezGómez, Phys. Rev. D88, 084042 (2013), arXiv:1308.5789 [gr-qc].

[13] M. Krššák, R. J. van den Hoogen, J. G. Pereira, C. G. Böhmer, and A. A. Coley, Class. Quant. Grav. 36, 183001 (2019), arXiv:1810.12932 [gr-qc].

[14] M. Fontanini, E. Huguet, and M. Le Del-

tions, even local, should not be confused, in GR, with the "local displacements" generated by the group of local diffeomorphisms, or more precisely, its Lie algebra, sometimes also referred as to liou, arXiv:1811.03810 [gr-qc].

[15] J. G. Pereira and Y. N. Obukhov, Proceedings, Teleparallel Universes in Salamanca: Salamanca, Spain, November 26-28, 2018, Universe 5, 139 (2019), arXiv:1906.06287 [gr-qc].

[16] M. Le Delliou, E. Huguet, and M. Fontanini, Phys. Rev. D 101, 024059 (2020), arXiv:1910.08471 [gr-qc].

[17] Fecko, M., Differential Geometry and Lie Groups for Physicists (Cambridge University Press, 2006).

[18] C. J. Isham, Modern differential geometry for physicists (World Scientific, Singapore, 1999).

[19] M. Nakahara, Geometry, topology and physics, 2nd ed. (CRC press, 2003).

[20] S. Kobayashi and K. Nomizu, Foundations of Differential Geometry, vol 1 (John Willey \& Sons, New York, 1963).

[21] D. K. Wise, Classical and Quantum Gravity 27, 155010 (2010).

[22] G. Catren, Int. J. Geom. Meth. Mod. Phys. 12, 1530002 (2015), arXiv:1407.7814 [gr-qc].

[23] R. Sharpe, Differential Geometry: Cartan's Generalization of Klein's Erlangen Program (Springer, New York, 1997).

[24] E. Huguet, M. L. Delliou, M. Fontanini, and Z. C. Lin, "Teleparallel gravity as a gauge theory: coupling to matter with cartan connection," (2020), arXiv:2008.13493 [gr-qc].

[25] D. Bleecker, Gauge theories and variational principles (Dover, 2005).

[26] M. Blagojević and F. W. Hehl, eds., Gauge Theories of Gravitation (World Scientific, Singapore, 2013).

[27] F. W. Hehl and Y. N. Obukhov, "Conservation of energymomentum of matter as the basis for the gauge theory of gravitation," (2020), arXiv:1909.01791 [gr-qc].

[28] J. François, S. Lazzarini, and T. Masson, "Gauge field theories: various mathematical approaches," (2014), arXiv:1404.4604 [math-ph]. 